\title{
Cross-Layer, Energy-efficient Design for Supporting Continuous Queries in Wireless Sensor Networks: A Quorum-Based Approach
}

\author{
Chia-Hung Tsai, Tsu-Wen Hsu, Meng-Shiuan Pan, and Yu-Chee Tseng \\ Department of Computer Science \\ National Chiao Tung University \\ Hsin-Chu, 30050, Taiwan \\ Email:\{chiahung, tsuwen, mspan, yctseng\}@cs.nctu.edu.tw
}

\begin{abstract}
Power saving and query processing are two major concerns in a wireless sensor network. Each of these two issues has been intensively studied separately in the literature. In this work, we are interested in linking the asynchronous power-saving protocol and the continuous query-processing problem together. A cross-layer solution is proposed. On the MAC layer, we propose to use the grid-quorum system [25] to serve as the underlying power-saving framework. On the network layer, we propose to find query paths based on the power cost incurred by grid quorums used by nodes along a path. We show how these two layers interwork with each other to support continuous queries in an energy-efficient way.
\end{abstract}

Keywords: power saving, protocol design, query processing, routing, wireless sensor network.

\section{Introduction}

The rapid progress of wireless communication and MEMS technology have made wireless sensor networks (WSNs) possible. A WSN normally consists of many inexpensive wireless sensor nodes. Each node is capable of collecting, storing, processing environmental information, and communicating with neighbor nodes. Recently, a lot of research works have been dedicated to WSNs, such as routing [6][9], self-organization [12][23], deployment 
[8][16][28], and localization [5][20]. Applications of WSNs include emergency guiding [13][26], light control [17][18], and environment monitoring [24].

Power saving and query processing are two main issues in WSNs. Many power-saving MAC protocols have been proposed. In SMAC [30], nodes periodically switch to sleep mode. In PMAC [31], sensors are allowed to adaptively determine their sleep schedules by considering neighbors' traffic patterns. In RMAC [3], sensor nodes periodically wake up and use their active periods to establish routing paths. Nodes not located on any routing path can go to sleep; otherwise, they have to remain active. GAF [29] divides the network area into square grids. Although sensors can switch between sleep mode and active mode periodically, it guarantees that at least one node per grid remains active to exchange packets with neighboring grids. Span [2] adaptively elects some nodes to stay in active mode and serves as the network backbone. Other nodes periodically check with backbone nodes to see if they need to wake up. Both [2] and [29] may have some redundant sensors to stay active. TAP [7] considers traffic flows and identifies redundant nodes that can go to sleep when establishing routing paths. Most of these schemes require nodes to be synchronized in time, which is costly. Recently, some power-saving protocols have been proposed without requiring time synchronization [1][11][14][25].

On the other hand, query processing in WSNs has also attracted a lot of attention. Directed diffusion [10] achieves energy efficiency by selecting empirically good paths and by caching and processing data inside the network. In [19], data-centric storage is proposed by adopting geographic hashing to offer high data availability and load distribution. TAG [15] is a tiny data service that can significantly reduce bandwidth consumption. A semistructure approach which uses multiple shortest-path trees is proposed in [4] to support scalable data aggregation. A lot of works [21][22] utilize the spatio-temporal correlations of sensing data to achieve energy efficiency. A generic two-tier storage strategy for answering precision-constrained approximate queries is proposed in [27]. Although most of these query-processing works focus on achieving energy efficiency, they all do not specifically address the underlying wake-up/sleep schedules of sensor nodes.

In this work, we are interested in applying the quorum-based power-saving protocols 
[1][11][14][25], which have the advantage of not relying on any time synchronization among sensor nodes, to the continuous query-processing problem. A continuous query involves sending periodical reports from a source to a sink and is commonly seen in WSNs. More specifically, we will adopt the grid-quorum system [25] to derive the wake-up/sleep schedules of sensor nodes. Multiple query paths may coexist, each with its preferred grid quorum. We will show how these paths (and thus grid quorums) interact with each other to meet each query's bandwidth requirement in an energy-efficient way. Although global clock synchronization is not necessary, we will suggest to employ an optional local slot synchronization to improve nodes' energy efficiency. Compared to existing works, this paper contributes in proposing a cross-layer approach to integrating the grid-quorum system with continuous queries. Simulation results are presented to evaluate our results.

The rest of this paper is organized as follows. Section 2 presents our cross-layer system architecture. The detail MAC layer (quorum layer) and network layer (query-processing layer) are presented in Section 3 and Section 4, respectively. Section 5 contains our simulation results. Finally, Section 6 concludes this paper.

\section{System Architecture}

We are given a WSN for supporting continuous queries. A continuous query is a unicast with sensing data being periodically delivered from a source node to a sink node. A continuous query, or simply query, is denoted by a 5-tuple $\left(s_{n}, s_{r}, t, p, l e n\right)$, where $s_{n}$ is the sink node, $s_{r}$ is the source node, $t$ is the lifetime of the query, $p$ is the period that $s_{r}$ will generate reports, and len is the expected packet length per report. Multiple queries may coexist in the network. We use the grid-quorum system [25] as the underlying MAC layer to support power management and develop a routing layer on the top of the quorum system to determine its parameters. The goal is to support continuous queries in an energy-efficient manner.

We propose a 2-layer architecture as shown in Fig. 1. When a continuous query arrives at the network layer, the sink will broadcast a query request ( $Q R E Q)$ packet to find a reporting path to the source. Such QREQ packets will be flooded around the network. To reply, the source will unicast a query reply $(Q R E P)$ packet to the sink. To save sensor nodes' energy, 


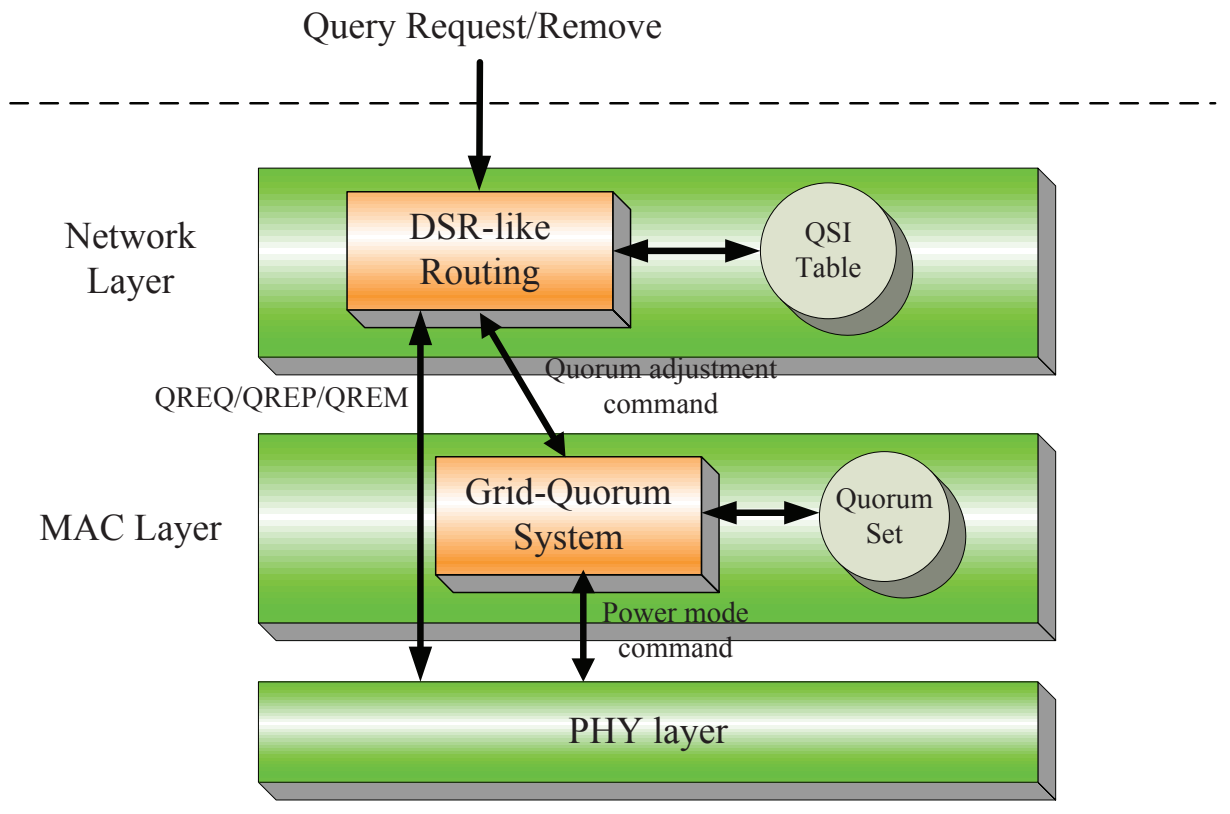

Figure 1: The proposed 2-layer architecture.

a cost function is designed at the network layer to select query paths and to dynamically choose/adjust the quorum system's parameters. Then, the MAC layer will give power mode commands to the underlying layer. Note that when there are multiple queries, our cross-layer approach will try to increase the overlapping among nodes' quorums to reduce the energy costs to support these queries. After a query expires, a query remove (QREM) packet will be sent along its query path.

Each node will maintain a Query Session Information (QSI) table to keep track of the query paths that currently pass it and the quorums to support these paths. Table 1 shows the structure of the QSI table. Gird quorums in this table will together form the quorum set of the node. The detail MAC-layer and network-layer operations will be discussed in Section 3 and Section 4, respectively. 
Table 1: An example of the QSI table.

\begin{tabular}{|c|c|c|c|c|}
\hline Query & Up_Node & Down_Node & Quorum & Additional_Quorum \\
\hline$(31,99,2000,40,100)$ & 55 & 129 & $(8,5,\{1\},\{1\})$ & $\phi$ \\
\hline$(101,29,1000,20,100)$ & 63 & 129 & $(5,4,\{3\},\{3\})$ & $\phi$ \\
\hline
\end{tabular}

(a)

(b)

$\mathrm{g}_{1}=(4,3,\{1\},\{1\})$

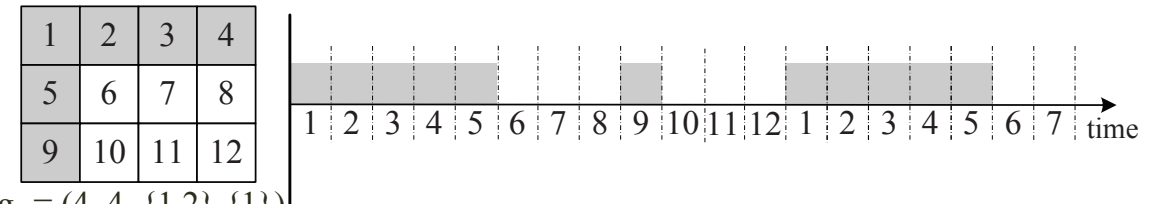

$\mathrm{g}_{2}=(4,4,\{1,2\},\{1\})$

\begin{tabular}{|c|c|c|c|}
\hline 1 & 2 & 3 & 4 \\
\hline 5 & 6 & 7 & 8 \\
\hline 9 & 10 & 11 & 12 \\
\hline 13 & 14 & 15 & 16 \\
\hline
\end{tabular}

$\mathrm{G}(\mathrm{v})=\left\{\mathrm{g}_{1}, \mathrm{~g}_{2}\right\}$

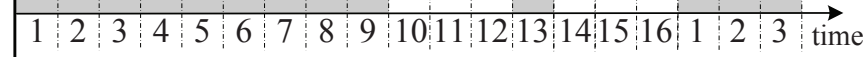

(c)

\begin{tabular}{|c|c|c|c|}
\hline 1 & 2 & 3 & 4 \\
\hline 5 & 6 & 7 & 8 \\
\hline 9 & 10 & 11 & 12 \\
\hline
\end{tabular}

\begin{tabular}{|c|c|c|c|}
\hline 1 & 2 & 3 & 4 \\
\hline 5 & 6 & 7 & 8 \\
\hline 9 & 10 & 11 & 12 \\
\hline 13 & 14 & 15 & 16 \\
\hline
\end{tabular}

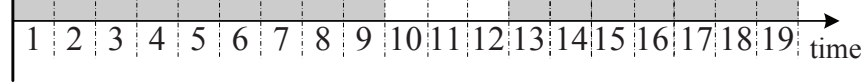

$\square$ : quorum slot (active)

$\square$ : non-quorum slot (sleep)

Figure 2: An example of a quorum set. 


\section{Quorum Layer}

\subsection{Grid Quorum System}

Power-saving protocols for wireless networks need to ensure that nodes' wakeup patterns will overlap with their neighbors' patterns for communication opportunity. It is pointed out in [25] that two major challenges that one would encounter when designing a power-saving protocol are: clock synchronization and neighbor discovery. Therefore, many solutions try to enforce nodes to synchronize their clocks. However, time synchronization in a large-scale distributed environment is very costly. An alternative is to develop asynchronous powersaving protocols. The quorum-based protocols [1][11][14][25] are such solutions. Basically, they require nodes to wake up and sleep based on some pre-configured rules, but nodes do not need to synchronize their clocks. Several kinds of quorums have been proposed, such as tree quorums and grid quorums.

In this work, we will adopt the grid-quorum system [25] as our power-saving mechanism. Fig. 2(a) shows a grid-quorum example. Each node's time axis is divided into repetitive $n_{1} \times n_{2}$ time slots, which are called a group. In each group, its slots are arranged as an $n_{1} \times n_{2}$ array in a row-major manner. From the array, the node can arbitrarily pick one column and one row of slots as its wakeup slots, or called quorum slots. Each node must stay awake in quorum slots, and can go to sleep in the remaining $n_{1} \times n_{2}-n_{1}-n_{2}+1$ slots. Note that nodes' clocks do not need to be synchronized.

The concept has been applied to IEEE 802.11-based ad hoc networks in [25] by enforcing all nodes to take the same values of $n_{1}$ and $n_{2}$. In [1], it is further shown that even if two nodes use different $n_{1}$ and $n_{2}$, transmission opportunity (i.e., overlapping of wake-up patterns) between them is still guaranteed.

\subsection{Quorum Set for Continuous Queries}

In this work, we are interested in applying the grid-quorum system to support continuous queries in a WSN. The wake-up/sleep schedule of a node will be determined by one or multiple grid quorums, which we call quorum set. The quorum set of a node $v$ is denoted 
by $G(v)$. Each grid quorum is denoted by a 4-tuple $g=\left(n_{1}, n_{2}, R, C\right)$, where $n_{1}$ and $n_{2}$ are the numbers of rows and columns, respectively, of the grid array, $R$ is a set of rows, and $C$ is a set of columns. Note that this is an extension of the original definition in [25] since all entries falling in rows of $R$ or columns of $C$ are quorum slots. We define the duty cycle of a grid quorum $g=\left(n_{1}, n_{2}, R, C\right)$ by

$$
\operatorname{dty}(g)=\frac{|R| \times n_{1}+|C| \times n_{2}-|R| \times|C|}{n_{1} \times n_{2}} .
$$

For example, Fig. 2(a) shows a grid quorum $g_{1}$ following the original definition of [25] (it contains only one row and one column of quorum slots). In Fig. 2(b), $g_{2}=(4,4,\{1,2\}$, $\{1\})$ is an extended grid quorum, which contains two rows and one column of quorum slots. Fig. 2(c) shows a quorum set $G(v)=\left\{g_{1}, g_{2}\right\}$, in which case, $v$ will run both quorums $g_{1}$ and $g_{2}$ simultaneously by "OR" the quorum slots of both $g_{1}$ and $g_{2}$. That is, whenever any of the grid quorums in $G(v)$ indicates that a slot is a quorum slot, $v$ will enter the active mode. So Fig. 2(c) is the "OR" of the two sequences in Fig. 2(a) and Fig. 2(b).

\section{Query-Processing Layer}

In our system, when a node does not support any continuous query, its quorum set will contain only one default grid quorum $g_{d e f}$ with minimum duty cycle. As more and more continuous queries (query paths) pass the node, its quorum set will contain more grid quorums. The default quorum is defined as $g_{\text {def }}=\left(n_{\max }, n_{\max },\{r n d\},\{r n d\}\right)$, where $n_{\max }$ is a large number and $r n d$ is a random integer between 1 and $n_{\max }$. A DSR-like routing protocol will be applied. To select a routing path, an energy cost function will be defined to evaluate the quality of a query path. Basically, a new path will try to increase its overlapping of quorum slots with existing paths' quorum slots while maintain sufficient communication capacity.

Section 4.1 presents the query-requesting process, followed by the query-replying and the query-removing processes in Section 4.2 and Section 4.3. Finally, in Section 4.4, a lightweight local slot synchronization is proposed to increase energy efficiency. 


\subsection{Query-Requesting Process}

This part contains three modules, quorum preparing, QREQ initiating and processing, and $Q R E Q$ rebroadcasting, as explained below.

A) Quorum Preparing: When a sink node $s_{n}$ has a query $y=\left(s_{n}, s_{r}, t, p\right.$, len $)$ to a source node $s_{r}$, it will compute a grid quorum $g_{i n i}$ to support the query $y$ as follows. Here we assume that from past history, the length len per report is already known.

1. Compute a pair $\left(n_{1}, n_{2}\right)$ such that $n_{1} \times n_{2} \approx p$ and $n_{1}$ is as close to $n_{2}$ as possible.

2. Construct a grid quorum $g_{i n i}=\left(n_{1}, n_{2}, R, C\right)$, where $R / C$ contains a random row/column.

3. Then we check whether $d t y\left(g_{\text {ini }}\right) \geq \frac{\text { len }}{r} \cdot \frac{1}{p}$ holds, where $r$ is the transmission rate of a node. If so, we will adopt $g_{i n i}$ as the grid quorum to serve the query $y$. Otherwise, we will continuously add rows or columns to $R$ or $C$ to increase the duty cycle value $\operatorname{dty}\left(g_{\text {ini }}\right)$, until $d t y\left(g_{\text {ini }}\right) \geq \frac{\text { len }}{r} \cdot \frac{1}{p}$ holds.

Note that $g_{i n i}$ is only considered as a candidate to support $y$; it may or may not be actually used on the query path between $s_{r}$ and $s_{n}$. This will become clear later.

B) QREQ Initiating and Processing: There are two cases involving in producing a QREQ packet: (i) a node initiates a new query and (ii) a node receives a QREQ and rebroadcasts it. Below, we will only consider case (ii) and regard case (i) as a special case of case (ii). So we suppose that node $x_{i}$ receives from node $x_{i-1}$ a $\mathrm{QREQ}\left(g_{i n i}, y, c, P A T H\right)$ for possibly supporting a query $y$ initiated by node $x_{0}$, where $g_{i n i}$ is the grid quorum computed by $x_{0}$ (by the above step A), $c$ is the cost calculated by $x_{i-1}$, and PATH is a list of 2-tuples, where each 2-tuple is of the form (node_id, quorum). Note that PATH contains the nodes that the QREQ has traversed so far and the grid quorums chosen by them. In case that $x_{i}$ is the query initiator (i.e., $x_{0}=x_{i}$ ), we will imagine that a virtual QREQ is sent by $x_{i}$ to itself such that $c=0$ and $P A T H=()$ is an empty list. On receipt such a QREQ, the following discusses how $x_{i}$ rebroadcasts this QREQ.

First, $x_{i}$ will find a quorum to serve query $y$, which we call $g_{s e r}(y)$. If $x_{i}$ is not currently passed by any query path, it will set $g_{s e r}(y)=g_{i n i}$. Otherwise, $x_{i}$ will try to pick an existing 
quorum in its quorum set $G\left(x_{i}\right)$ or adopt $g_{i n i}$ to serve $y$. It will try to pick an existing one in $G\left(x_{i}\right)$ first. Recall the definition of duty cycle in Eq. (1). Given $G\left(x_{i}\right)$, we can estimate $x_{i}$ 's duty cycle as follows:

$$
\operatorname{DTY}\left(G\left(x_{i}\right)\right)=1-\prod_{g \in G\left(x_{i}\right)}(1-\operatorname{dty}(g)) .
$$

Also, from $x_{i}$ 's QSI, we can measure $x_{i}$ 's current traffic load as follows. For each query $z$, in $x_{i}$ 's QSI, its load can be calculated by $l d(z)=\frac{\operatorname{len}(z)}{r} \cdot \frac{1}{p(z)}$, where $l e n(z)$ is the length of each sensing report and $p(z)$ is the period per report for query $z$. So $x_{i}$ 's current traffic load is

$$
L D\left(x_{i}\right)=\sum_{\forall z \in Q S I \text { of } x_{i}} l d(z) .
$$

Then, $x_{i}$ can measure whether its current quorum set can accommodate $y$ or not by checking $L D\left(x_{i}\right)+l d(y) \leq D T Y\left(G\left(x_{i}\right)\right)$. If so, $x_{i}$ will try to pick a candidate quorum $g_{\text {can }} \in G\left(x_{i}\right)$ with sufficient capacity to serve $y$. The capacity of $g_{\text {can }}$ is defined as follows:

$$
\operatorname{Cap}\left(g_{c a n}\right)=\frac{\sum_{s_{j} \in Q S\left(g_{c a n}\right)} \frac{1}{s-\operatorname{deg}\left(s_{j}\right)}}{n_{1}\left(g_{c a n}\right) \times n_{2}\left(g_{c a n}\right)},
$$

where $n_{1}\left(g_{c a n}\right)$ and $n_{2}\left(g_{c a n}\right)$ are the numbers of rows and columns of $g_{c a n}$, respectively, $Q S\left(g_{c a n}\right)$ means the set of quorum slots of $g_{c a n}$, and $s-\operatorname{deg}\left(s_{j}\right)$ is the share degree of the quorum slot $s_{j}$ in $g_{c a n}$. Here the share degree of $s_{j}$ is the estimated average number of quorums which will also regard slot $s_{j}$ as a quorum slot. This is due to the fact that $x_{i}$ may be running several quorums simultaneously to support multiple query paths, so quorum $g_{\text {can }}$ can only have an equal share of that slot. (For example, in Fig. 2, the share degree of slot 5 of $g_{2}$ is two and the share degree of slot 6 of $g_{2}$ is one.) If there exists one $g_{\text {can }}$ such that

$$
\operatorname{Cap}\left(g_{\text {can }}\right) \geq l d(y)+\sum_{z \text { supported by } g_{\text {can }}} l d(z),
$$

then $g_{\text {can }}$ will be assigned to support $y$ and we will set $g_{s e r}(y)=g_{\text {can }}$. Otherwise, no existing quorum in $G\left(x_{i}\right)$ can support $y$ and we will check the following two conditions to see if it is possible to include $g_{\text {ini }}$ into $G\left(x_{i}\right)$ : 
- $D T Y\left(G\left(x_{i}\right) \cup\left\{g_{i n i}\right\}\right) \geq L D\left(x_{i}\right)+l d(y)$

- $C a p\left(g_{i n i}\right) \geq l d(y)$

If both conditions are met, we will set $g_{s e r}(y)=g_{\text {ini }}$; otherwise, this query is beyond the capacity of $x_{i}$ to be supported and the QREQ will be discarded. Finally, if $y$ can be supported, $x_{i}$ will append the 2-tuple $\left(x_{i}, g_{\text {ser }}(y)\right)$ to the list $P A T H$ and proceed to the next step.

The above steps have determined the quorum $g_{s e r}(y)$ to support $y$. Next, we will compute the additional energy cost to support $y$. There are two costs associated with this: (i) the average extra energy cost $C_{a c t}$ for $x_{i}$ to remain active per slot and (ii) the average extra energy $\operatorname{cost} C_{t x}$ for $x_{i}$ to transmit data for $y$ per slot. For (i), recall that $g_{s e r}(y)$ is the quorum to serve $y$ by $x_{i}$. Let $g_{s e r}^{\prime}(y)$ be the quorum selected by $x_{i-1}$ to serve $y$. We will actually enforce $x_{i}$ to include $g_{s e r}^{\prime}(y)$ into its quorum set, so that $x_{i}$ can smoothly transmit data to $x_{i-1}$. The cost $C_{a c t}$ is defined as

$$
C_{a c t}=E_{a c t} \times\left(D T Y\left(G\left(x_{i}\right) \cup\left\{g_{\text {ser }}(y), g_{\text {ser }}^{\prime}(y)\right\}\right)-D T Y\left(G\left(x_{i}\right)\right)\right),
$$

where $E_{\text {act }}$ is the energy to remain active for one full slot. This means the extra amount of energy for $x_{i}$ to remain active per slot in order to support $y$. For (ii), the cost $C_{t x}$ is defined as

$$
C_{t x}=\left(E_{t x}-E_{a c t}\right) \times \frac{\operatorname{len}(y)}{r} \times \frac{1}{p(y)},
$$

where $E_{t x}$ is the energy to transmit one full slot of data.

The total addition energy cost for $x_{i}$ to support $y$ is $C_{a c t}+C_{t x}$. So we will set $c=$ $c+C_{a c t}+C_{t x}$.

C) QREQ Rebroadcasting: The above steps have determined the new $c$ and PATH if $x_{i}$ decides to support $y$. Node $x_{i}$ will also maintain the minimum $\operatorname{cost} c_{\min }$ for all paths from $x_{0}$ to $x_{i}$ that $x_{i}$ has learned so far. If $c_{\text {min }} \geq c$, then $x_{i}$ will rebroadcast $\operatorname{QREQ}\left(g_{i n i}, y, c\right.$, $P A T H$ ) containing the new $c$ and $P A T H$ and set $c_{\min }=c$. Note that in cast that $x_{i}$ is the source $s_{r}$, rebroadcasting QREQ is not necessary (this will be discussed in Section 4.2). 


\subsection{Query-Replying Process}

When a node $x_{i}$ receives from $x_{i-1}$ a QREQ $\left(g_{i n i}, y, c, P A T H\right)$ initiated by a node $x_{0}$ and finds that it is the sink node of the query $y$, it will prepare to periodically report its sensing data to $x_{0}$ according to the parameters specified in the query. Node $x_{i}$ will collect QREQs for a while and choose the $\operatorname{QREQ}\left(g_{i n i}, y, c, P A T H\right)$ with the lowest cost $c$. Then $x_{i}$ will unicast $\mathrm{QREP}(y, P A T H)$ back to $x_{0}$. The $\mathrm{QREP}$ will sequentially traverse nodes along the reverse direction of $P A T H$.

For each node $x_{j}$ receiving the QREP, it can identify its serving quorum $g_{\text {ser }}(y)$ recorded in the PATH. There are two cases:

- If $G\left(x_{j}\right)=\left\{g_{d e f}\right\}, x_{j}$ will directly set $G\left(x_{j}\right)=\left\{g_{\text {ser }}(y)\right\}$.

- Otherwise, $x_{j}$ will set $G\left(x_{j}\right)=G\left(x_{j}\right) \cup\left\{g_{s e r}(y)\right\}$.

Also, $x_{j}$ can find the serving quorum, say $g_{s e r}^{\prime}(y)$, picked by its previous node in $P A T H$. If $g_{\text {ser }}(y) \neq g_{\text {ser }}^{\prime}(y), x_{j}$ will further set $G\left(x_{j}\right)=G\left(x_{j}\right) \cup\left\{g_{\text {ser }}^{\prime}(y)\right\}$. This is for $x_{j}$ to cooperate with its previous node so as to smoothly transmit its data to its previous node. Finally, $x_{j}$ will adjust its QSI table as follows (refer to Table 1). A new entry will be added such that Query $=y, \operatorname{Up} \_$Node $=x_{j}$ 's previous node, Down $\_$Node $=x_{j}$ 's next node, Quorum $=g_{s e r}(y)$, and Additional_Quorum $=g_{\text {ser }}^{\prime}(y)$.

After a node adjusts its quorum set, it can wake up and sleep according to the quorums in its set. Quorums do not need to synchronize with each other. Whenever any quorum in its set enters a quorum slot, the node has to be active in that slot. Also note that when a quorum slot belongs to multiple queries, the transmission opportunity should be equally shared by all these queries.

\subsection{Query-Removing Process}

When a query session $y$ terminates, the sink node can identify this fact by checking its QSI table. Then it can initiate a $Q R E M(y)$ packet along the query path to the sink. Each intermediate node when receiving the $Q R E M(y)$ will remove the corresponding entry from its 
QSI table. Also, the corresponding quorums to support will be removed from their quorum slots. Again, each node will wake up and sleep according to its new quorum slots.

\subsection{Local Slot Synchronization}

Although the quorum system can guarantee the communication opportunity of any two asynchronous nodes, in this section we will suggest a lightweight local slot synchronization to improve energy efficiency and reduce transmission delays of sensing reports. Here, we only propose to synchronize local nodes' slots and local nodes' quorums. We summarize our rules as follows:

- At the clock level, two neighboring nodes will try to synchronize their clocks by aligning their slots. That is, they will try to synchronize the beginning of slots at each side.

- At the quorum level, if two neighboring nodes use the same quorum in their quorum sets, they will try to synchronize this quorum by aligning the first slot of this quorum at each side. (Different quorums of these two nodes do not need to be synchronized. Similarly, inside each node, two different quorums do not need to be synchronized).

The above two rules do not address how to break the tie when a node has multiple neighbors and/or when a node shares the same quorum with multiple neighbors. We propose to assign priority by the following rules:

- Along a query path, a node that is closer to the source node has a higher priority.

- Between two query paths, the path which was established earlier (i.e., with an earlier timestamp) has a higher priority.

\section{Simulation Results}

\subsection{Simulation Environments}

Since large-scare deployment is difficult to realize, we develop a simulation environment to verify the energy efficiency factor of our cross-layer query-processing protocol. We set up 
a $400 \times 400 \mathrm{~m}^{2}$ sensing field, on which hundreds of sensor nodes are randomly deployed. The transmission range and carrier sensing range of each sensor node are set to 50 and 100 meters, respectively. In our simulations, we will randomly generate several sink-source continuous query pairs with random report periods and lifetimes. The whole simulation time is 7200 seconds. To evaluate the energy consumption, the power consumption rates of a wireless interface are set to 50,50, 45, and $5 \mathrm{~mW}$ under transmit, receive, idle, sleep modes, respectively. The default quorum $g_{d e f}$ is set to $(40,40,\{1\},\{1\})$ with each quorum slot fixed to 0.1 second. Hence, each node will initially operate under $5 \%$ duty cycle and each quorum group is 160 seconds.

Fig. 3 shows a scenario of our system which runs 4 continuous queries simultaneously. It shows that there exists path sharing between the sink-source pairs $\left(y_{1}, y_{1}^{\prime}\right)$ and $\left(y_{3}, y_{3}^{\prime}\right)$ from node 8 to node 131 , and the sink-source pairs $\left(y_{2}, y_{2}^{\prime}\right)$ and $\left(y_{4}, y_{4}^{\prime}\right)$ from node 148 to node 24. After the simulation terminates, the percentage of nodes' residual energy is displayed in Fig. 4.

In the following sections, we will discuss the benefit of our cross-layer design and the impact of query loads on our approach.

\subsection{Impact of Our Cross-Layer Design}

To verify the benefit gained from our cross-layer design, we will compare our approach against two schemes. Both schemes apply shortest path routing. The first one lets each query path adjust its quorum on this own, but there is no coordination between paths' quorums; this scheme is referred to as SP-NC (shortest-path, no-coordination). The second one enforces all quorum paths to share the same quorum; this scheme is referred to SP-GQ (shortest path, global-quorum). We show our results below.

A) Comparison with the SP-NC Scheme: Each query reporting period is set to 60 seconds. Query requests are randomly injected at a rate of one query per 500 seconds. Fig. 5 shows the minimal residual energy among all nodes. Since our scheme encourages a new path to overlap with existing paths, it shows that the SP-NC scheme is more likely to exhaust some particular nodes' energy. 


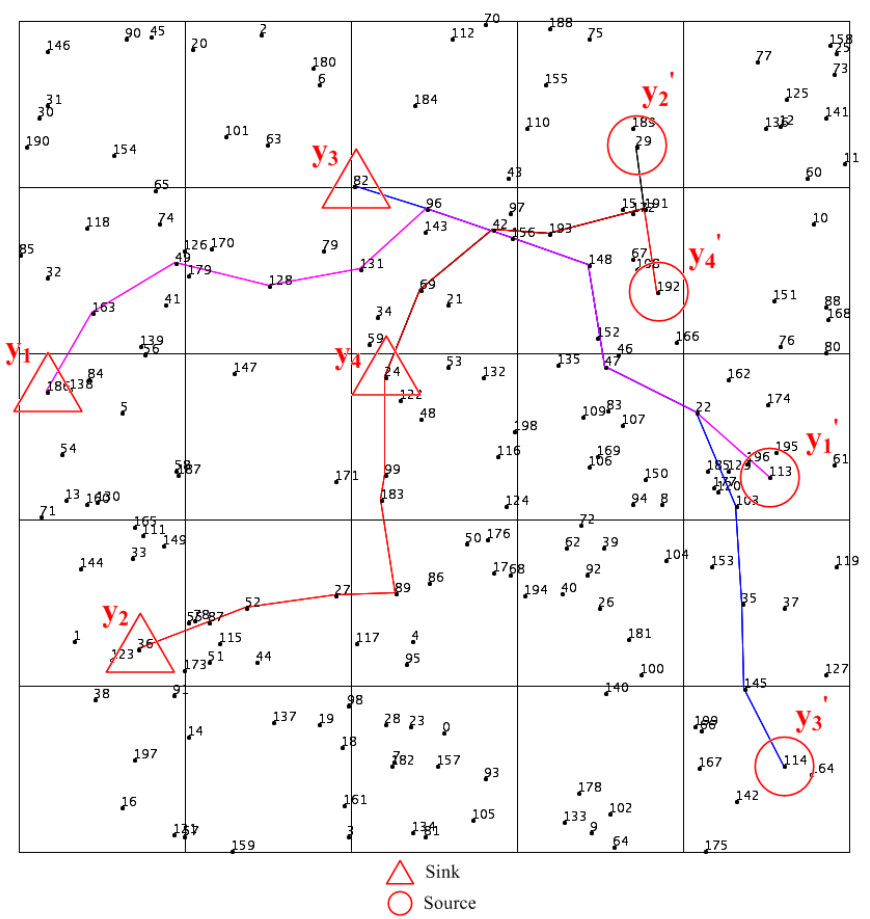

Figure 3: A path-sharing scenario.

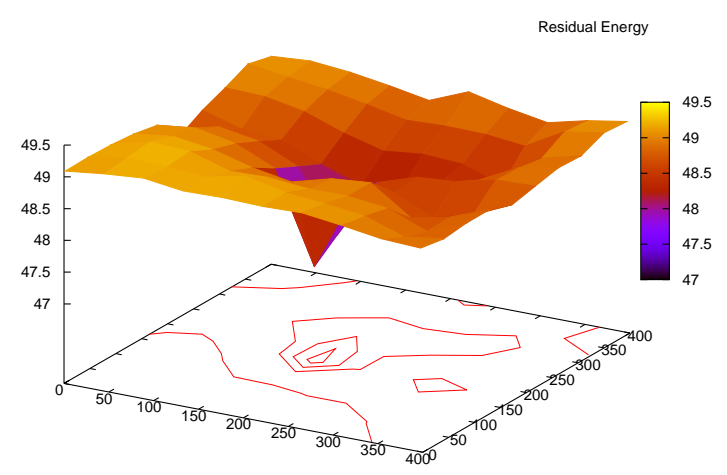

Figure 4: A scenario of the percentage of nodes' residual energy after executing 4 continuous queries. 


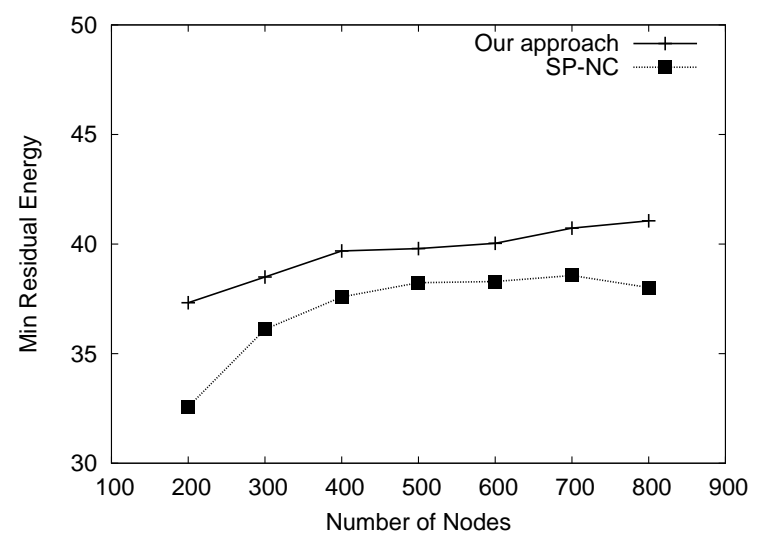

Figure 5: Comparison to the SP-NC scheme on minimal residual energy.

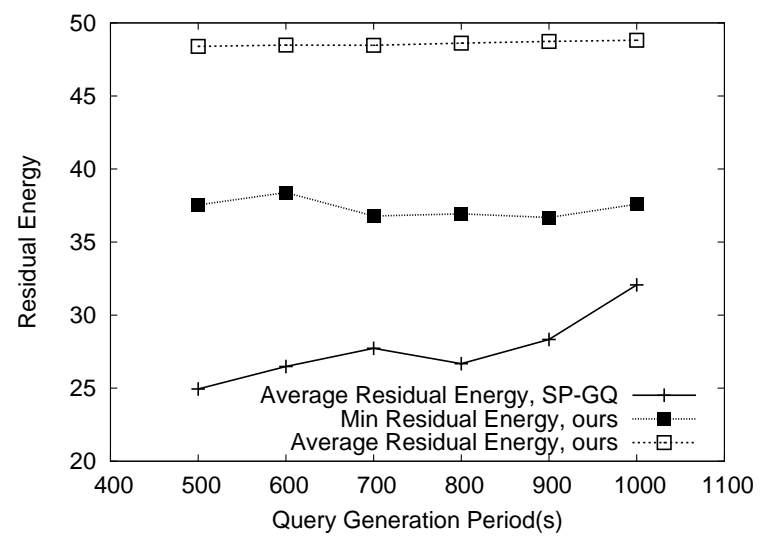

Figure 6: Comparison to the SP-GQ scheme on nodes' residual energy. 


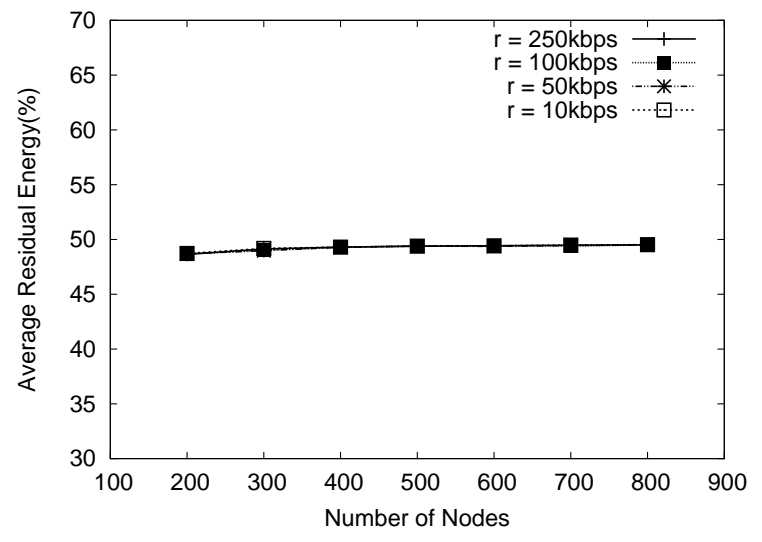

(a)

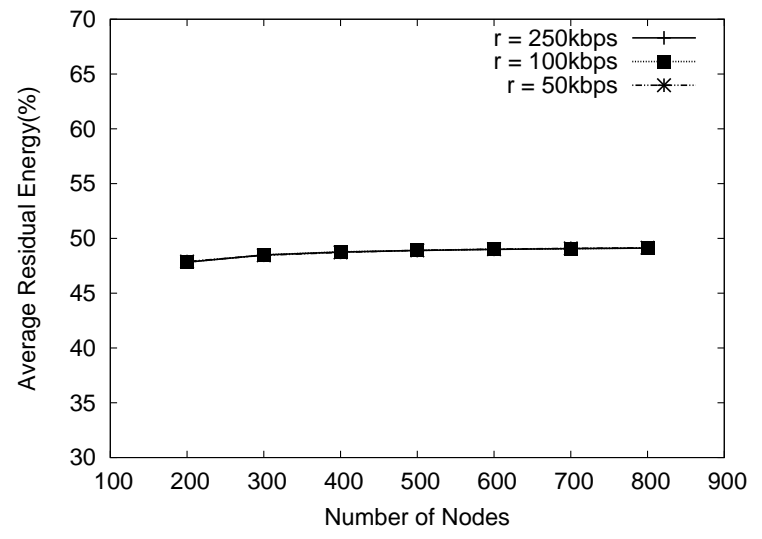

(c)

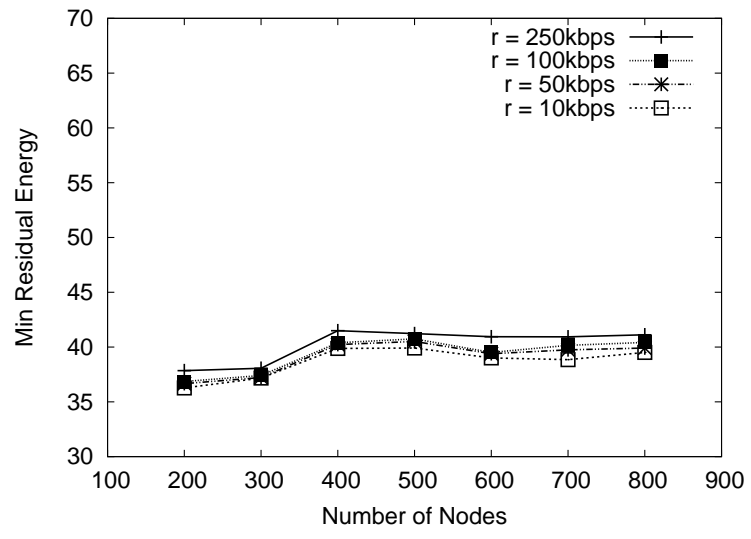

(b)

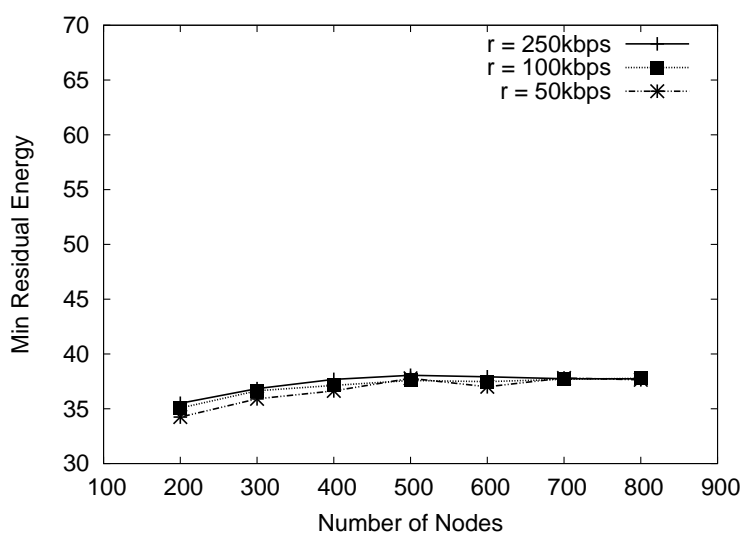

(d)

Figure 7: The energy consumption of our system under different transmission rates $(r)$.

B) Comparison with SP-GQ scheme: The SP-GQ scheme will pick the quorum with the lowest duty cycle that can meet all nodes' requirement as the global quorum. On the contrary, our scheme can dynamically adjust each query path's quorum. The results are in Fig. 6. We fix the number of nodes to 200 and set the query generation rate to one query per 500 seconds to 1000 seconds. It shows that our cross-layer design can result in much higher average residual energy. Even the minimum residual energy of our scheme still significantly outperforms that of SP-GQ. Also, the query generation rate has little impact on the energy consumption of our scheme. 


\subsection{Impact of Traffic Loads}

Recall the query load estimation in Section 4.1. It can be influenced by three factors: transmission rate, packet length per report, and reporting period. In the following, we will discuss the impact of traffic loads on energy consumption.

A) Impact of Transmission Rate: A smaller transmission rate $r$ will result in slower transmission (and thus a higher traffic load). Hence, we evaluate the energy consumption of our system by varying the transmission rate at $250 \mathrm{kbps}, 100 \mathrm{kbps}, 50 \mathrm{kbps}$, and $10 \mathrm{kbps}$. In Fig. 7(a)-(b), we randomly inject queries at a rate of one query per 100 seconds. In Fig. 7(c)(d), we randomly inject queries at a rate of three queries per 1000 seconds. Each report is 100 bytes. We can see that a lower $r$ might incur higher energy consumption. In Fig. 7(a) and Fig. 7(c), we see that both transmission rate and number of nodes make little impact on the average residual energy because our protocol only causes nodes on query paths to increase their duty cycles. All other nodes still operate with the default quorum. However, if we look at the node with the minimal residual energy, there do exist some differences, as shown in Fig. 7(b) and Fig. 7(d). A lower $r$ will cause some nodes to consume more energy than others but the impact is still quite smaller.

B) Impact of Packet Length: Here, we vary the length len per report to evaluate the energy performance of our scheme. The transmission rate $r$ is fixed to $250 \mathrm{kbps}$ and len varies from 100,1000 , to 5000 bytes. Similar with the previous case, the query generation rates are one and three queries per 1000 seconds in Fig. 8(a)-(b) and Fig. 8(c)-(d), respectively. Fig. 8(a) and Fig. 8(c) show that the average residual energy under different lens, while Fig. 8(b) and Fig. 8(d) show the minimal residual energy under different lens. The tread is generally the same as that in Fig. 7.

C) Impact of Query Period: In this scenario, we set $r=250 \mathrm{kbps}$ and len $=100$ bytes and vary the reporting period $p$ from 30 to 70 seconds. The query generation rates remain the same with the previous two experiments. The results are similar to the previous cases. As Fig. 9 shows, a higher reporting period will incur less energy consumption. From Fig. 9, we see that reporting period $(p)$ has more impact on energy consumption than transmission rate $(r)$ and packet length (len). This is because a lower reporting period will cause nodes to 


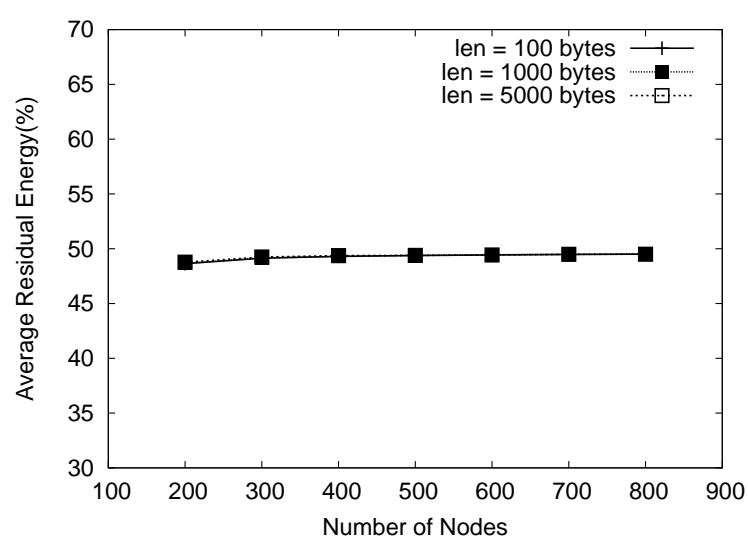

(a)

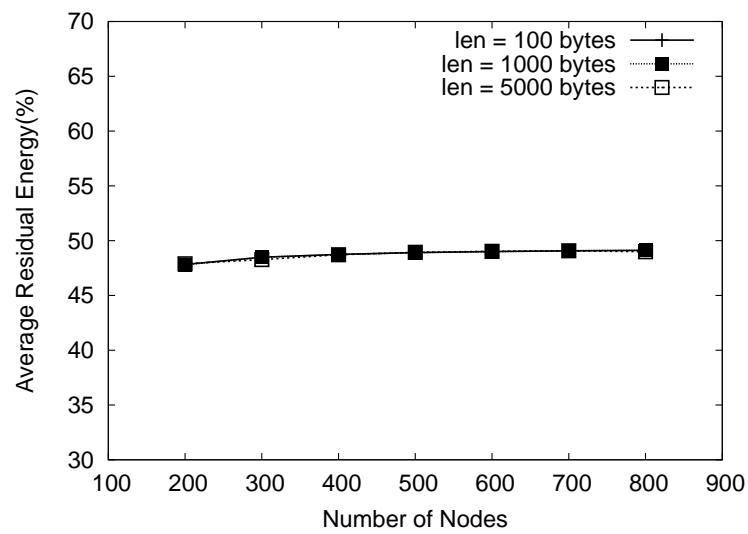

(c)

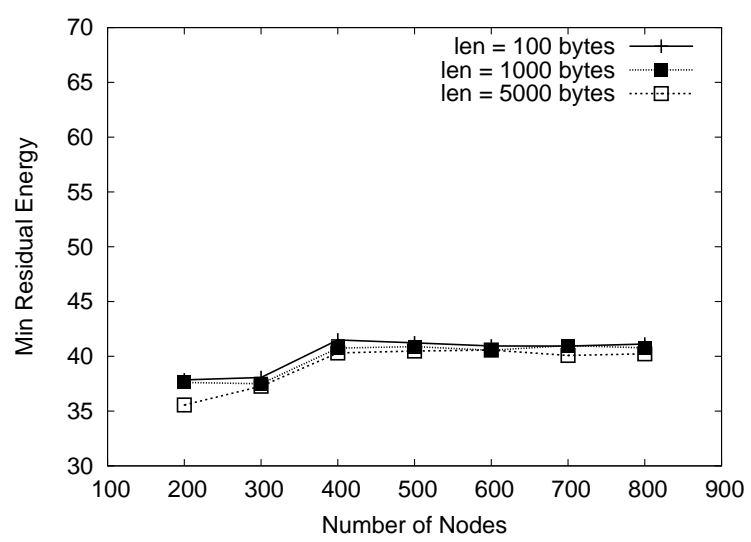

(b)

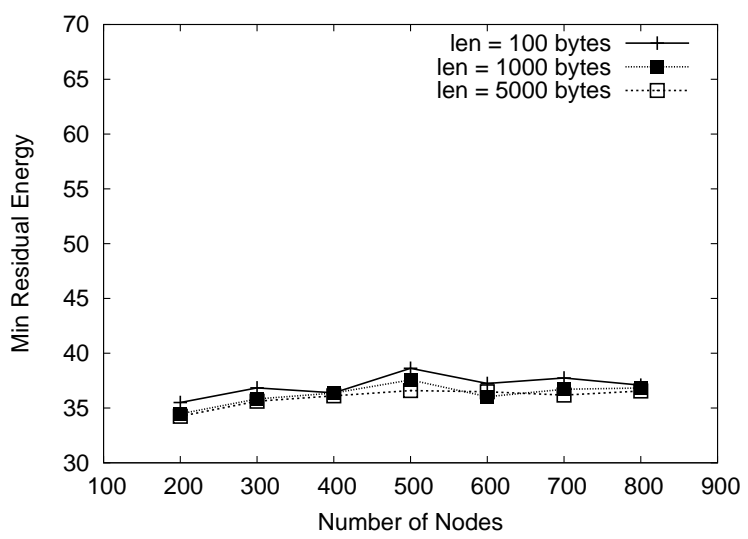

(d)

Figure 8: The energy consumption of our system under different lengths per report (len). 


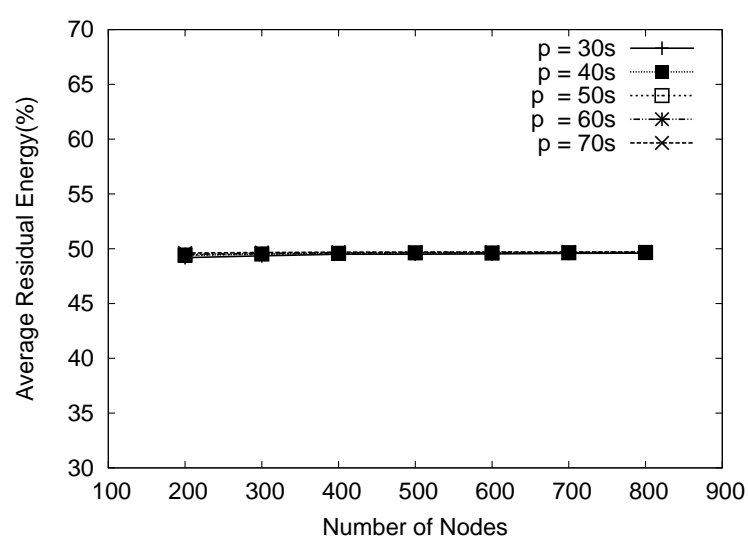

(a)

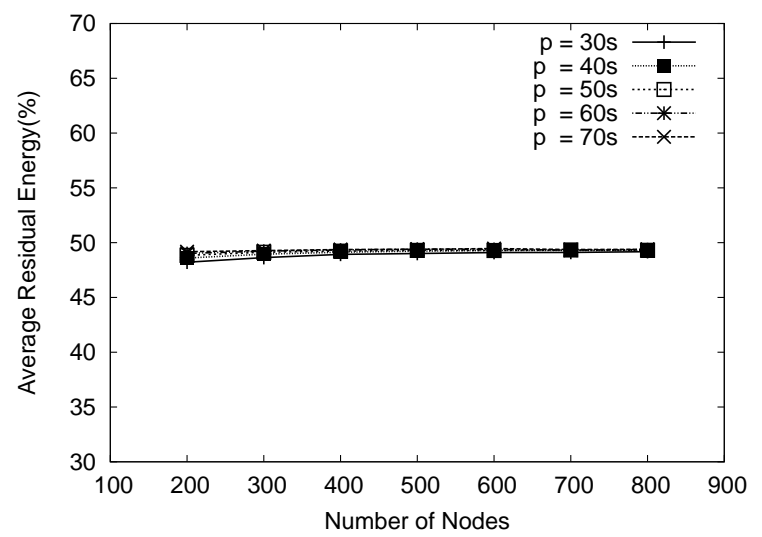

(c)

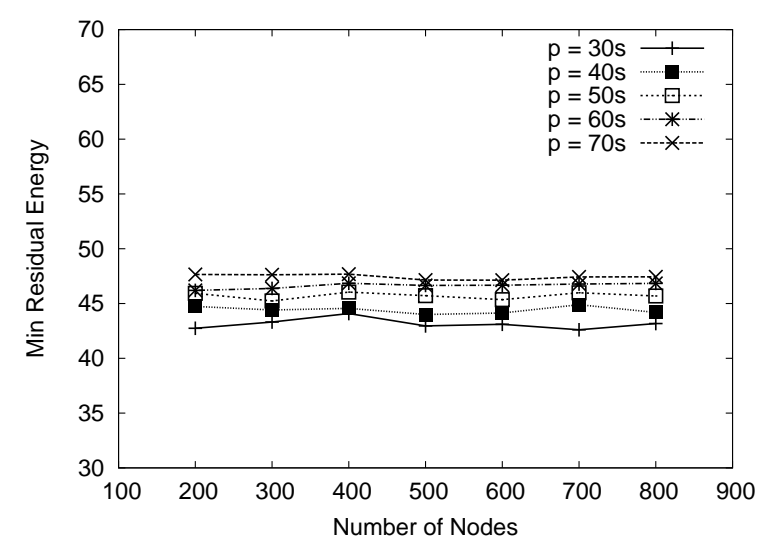

(b)

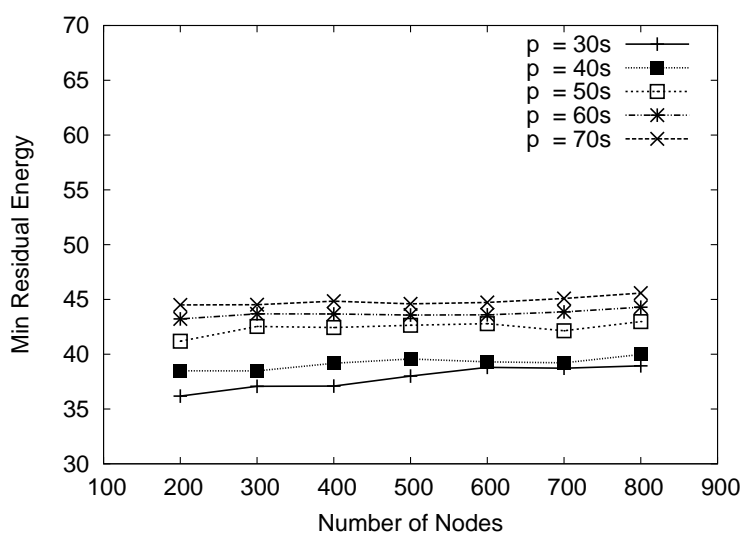

(d)

Figure 9: The energy consumption of our system under different reporting periods $(p)$. 
use smaller quorums to serve them. Smaller quorums can easily increase nodes' duty cycles.

\section{Conclusions}

We have developed a query-processing protocol to support multiple continuous queries simultaneously in a wireless sensor network. Our design emphasizes on increasing the overlapping of query paths for energy efficiency. It adopts the grid quorum system and extends it to the concept of quorum set. We modify the original DSR routing scheme by adding a cost metric to choose quorums along a query path. Simulation results also verify the correctness and performance of the proposed scheme. In the future, we will consider this issue in mobile WSNs.

\section{References}

[1] C.-M. Chao, J.-P. Sheu, and I.-C. Chou. An adaptive quorum-based energy conserving protocol for ieee 802.11 ad hoc networks. IEEE Trans. Mobile Computing, 5(5):560570, 2006.

[2] B. Chen, K. Jamieson, H. Balakrishnan, and R. Morris. Span: An energy-efficient coordination algorithm for topology maintenance in ad hoc wireless networks. In Proc. of ACM Int'l Conference on Mobile Computing and Networking (MobiCom), 2001.

[3] S. Du, A. K. Saha, and D. B. Johnson. Rmac: A routing-enhanced duty-cycle MAC protocol for wireless sensor networks. In Proc. of IEEE INFOCOM, 2007.

[4] K.-W. Fan, S. Liu, and P. Sinha. Dynamic forwarding over tree-on-dag for scalable data aggregation in sensor networks. IEEE Trans. Mobile Computing, 7(10):1271-1284, 2008.

[5] T. He, C. Huang, B. M. Blum, J. A. Stankovic, and T. Abdelzaher. Range-free localization schemes for large scale sensor networks. In Proc. of ACM Int'l Conference on Mobile Computing and Networking (MobiCom), pages 81-95, 2003.

[6] W. R. Heinzelman, A. Chandrakasan, and H. Balakrishnan. Energy-efficient communication protocols for wireless microsensor networks. In Proc. of Hawaii Int'l Conference on Systems Science (HICSS), 2000.

[7] P. Hu, P.-L. Hong, J.-S. Li, and Z.-Q. Qin. Tap: Traffic-aware topology control in on-demand ad hoc networks. Computer Networks, 29(18):3877-3885, 2006. 
[8] C.-F. Huang, Y.-C. Tseng, and L.-C. Lo. The coverage problem in three-dimensional wireless sensor networks. Journal of Interconnection Networks, 8(3):209-227, 2007.

[9] X.-M. Huang and J. Ma. Optimal distance geographic routing for energy efficient wireless sensor networks. International Journal of Ad Hoc and Ubiquitous Computing, 1(4):203-209, 2006.

[10] C. Intanagonwiwat, R. Govindan, D. Estrin, J. Heidemann, and F. Silva. Directed diffusion for wireless sensor networking. IEEE/ACM Trans. Networking, 11(1):2-16, 2003.

[11] J.-R. Jiang, Y.-C. Tseng, C.-S. Hsu, and T.-H. Lai. Quorum-based asynchronous powersaving protocols for ieee 802.11 ad hoc networks. ACM/Kluwer Mobile Networks and Applications, 10(1/2):169-181, 2005.

[12] M. Kochhal, L. Schwiebert, and S. Gupta. Role-based hierarchical self organization for wireless ad hoc sensor networks. In Proc. of ACM Int'l Workshop on Wireless Sensor Networks and Applications (WSNA), 2003.

[13] Q. Li, M. DeRosa, and D. Rus. Distributed algorithm for guiding navigation across a sensor network. In Proc. of ACM Int'l Symposium on Mobile Ad Hoc Networking and Computing (MobiHoc), Maryland, USA, 2003.

[14] W.-H. Liao, H.-H. Wang, and W.-C. Wu. An adaptive MAC protocol for wireless sensor networks. In Proc. of IEEE Int'l Symposium on Personal, Indoor and Mobile Radio Communications (PIMRC), 2007.

[15] S. Madden, S. Madden, M. J. Franklin, M. J. Franklin, J. Hellerstein, J. Hellerstein, W. Hong, and W. Hong. TAG: a tiny aggregation service for ad-hoc sensor networks. In Proc. of ACM Int'l Symposium on Operating Systems Design and Implementation, 2002.

[16] S. Meguerdichian, F. Koushanfar, M. Potkonjak, and M. B. Srivastava. Coverage problems in wireless ad-hoc sensor networks. In Proc. of IEEE INFOCOM, 2001.

[17] M.-S. Pan, L.-W. Yeh, Y.-A. Chen, Y.-H. Lin, and Y.-C. Tseng. A wsn-based intelligent light control system considering user activities and profiles. IEEE Sensors Journal, 8(10):1710-1721, 2008.

[18] H. Park, M. B. Srivastava, and J. Burke. Design and implementation of a wireless sensor network for intelligent light control. In Proc. of ACM/IEEE Int'l Conference on Information Processing in Sensor Networks (IPSN), 2007.

[19] S. Ratnasamy, B. Karp, S. Shenker, D. Estrin, R. Govindan, L. Yin, and F. Yu. Datacentric storage in sensornets with ght, a geographic hash table. Mobile Networks and Applications, 8(4):427-442, 2003. 
[20] A. Savvides, C.-C. Han, and M. B. Strivastava. Dynamic fine-grained localization in ad-hoc networks of sensors. In Proc. of ACM Int'l Conference on Mobile Computing and Networking (MobiCom), pages 166-179, 2001.

[21] P. Schaffer and I. Vajda. Cora: correlation-based resilient aggregation in sensor networks. In Proc. of ACM/IEEE Int'l Symposium on Modeling, Analysis and Simulation of Wireless and Mobile Systems (MSWiM), 2007.

[22] A. Skordylis, A. Guitton, and N. Trigoni. Correlation-based data dissemination in traffic monitoring sensor networks. In Proc. of IEEE Wireless Communications and Networking Conference (WCNC), 2006.

[23] K. Sohrabi, J. Gao, V. Ailawadhi, and G. J. Pottie. Protocols for self-organization of a wireless sensor network. IEEE Personal Communications, 7(5):16-27, October 2000.

[24] R. Szewczyk, A. Mainwaring, J. Polastre, J. Anderson, and D. Culler. An analysis of a large scale habitat monitoring application. In Proc. of ACM Int'l Conference on Embedded Networked Sensor Systems (SenSys), 2004.

[25] Y.-C. Tseng, C.-S. Hsu, and T.-Y. Hsieh. Power-saving protocols for ieee 802.11-based multi-hop ad hoc networks. Computer Networks, 43(3):317-337, 2003.

[26] Y.-C. Tseng, M.-S. Pan, and M.-S. Pan. A distributed emergency navigation algorithm for wireless sensor networks. IEEE Computer, 39(7):55-62, 2006.

[27] M. Wu, J. Xu, and X. Tang. Processing precision-constrained approximate queries in wireless sensor networks. In Proc. of ACM/IEEE Int'l Conference on Mobile Data Management, 2006.

[28] T.-T. Wu and K.-F. Ssu. Determining active sensor nodes for complete coverage without location information. International Journal of Ad Hoc and Ubiquitous Computing, 1(1/2):38-46, 2005.

[29] Y. Xu, J. Heidemann, and D. Estrin. Geography-informed energy conservation for ad hoc routing. In Proc. of ACM Int'l Conference on Mobile Computing and Networking (MobiCom), 2001.

[30] W. Ye, J. Heidemann, and D. Estrin. An energy-efficient MAC protocol for wireless sensor networks. In Proc. of IEEE INFOCOM, 2002.

[31] T. Zheng, S. Radhakrishnan, and V. Sarangan. Pmac: An adaptive energy-efficient MAC protocol for wireless sensor networks. In Proc. of IEEE Int'l Parallel and Distributed Processing Symposium (IPDPS), 2005. 